\title{
Preliminary Construction of the Behavior Spectrum of Cybister Japonicus Sharp under Artificial Breeding Conditions
}

\author{
Xiaoling Wang ${ }^{1, a}$, Tiefeng Wang ${ }^{1, a}$, Yilin Sun ${ }^{1, a}$, Shuang Wang ${ }^{1, a}$ \\ Yuan Cai ${ }^{1 \text {,a }}$, Yunkai Li ${ }^{1, a}$, Siyu Zhang ${ }^{1, a}$ \\ ${ }^{1}$ Baicheng Normal University, College of life science, BaiCheng, Jilin \\ abcwxl@126.com
}

Keywords: Artificial Breeding, Cybister Japonicus Sharp, Behavior Spectrum, Construction

\begin{abstract}
The 30 Cybister japonicus Sharp margin behavior were observed, recording, analysis and description in the laboratory of animal experiment center of Baicheng Normal University from 2016.3.6 to 2016.5.6 and 2016.5.7 to 2016.7.7 and 2016.7.17 to 2016.8.27, which lasted 160 days, a total of 640 hours. And the behavior spectrum of Cybister Japonicus Sharp was constructed. The construction of behavior spectrum would provide reference for behavior research and artificial breeding of Cybister Japonicussharp.
\end{abstract}

\section{Introduction}

The Cybister japonicus Sharp is an insect belonging to the Lsecta, the Coleoptera, and Dytiscidae. Has a wide distribution, northeast China, north China, central China, south China, east China are distributed. Screen is a lot of more phyletic, there are 2050 species around the world. The larger the Cybister japonicus Sharp is the southeast, the northeast region in China as a food and a traditional folk medicine aquatic insects, is a good treatment for kidney disease and special flavor, become a 21st century green food. So far about Cybister japonicus Sharp's morphological structure, culture, bionics and other aspects of the reports, and about the yellow edge water beetle behavior spectrum reported for the first of the part of the building, so the author of artificial breeding Cybister japonicus Sharpunder the condition of a systematic and comprehensive study, and constructs the yellow rim spanning the behavior of the spectrum, for the further research of Cybister japonicus Sharp and artificial breeding to provide the reference.

\section{Experimental Materials and Feeding Methods}

The Cybister japonicus Sharp of a fish pond in zhenlai county, jilin province, 30 heads. The food is small fish and chicken, rabbit, large fish to remove fat innards. Feed every day at 10:00-11:00am. Feeding is $7-8 \%$ of the average weight of the worm.

The experiment was done in the lab at the experimental center of baicheng normal university. The experimental temperature is the temperature of the laboratory. The container of the life of the Cybister japonicus Sharp is $30 \mathrm{~cm}$ in diameter and $15.5 \mathrm{~cm}$ high in the glass cistern.

Behavior observation is divided into three stages and record respectively, is the first time in March 2016 on May 6, 6 solstice, using random sampling method, using the random sampling method, observe the four times a day, every time 1 hour, observation time is 60 days. The second time period is 7 July 2016. The main target sampling method is to observe four times a day, one hour each time, and the observation time is 60 days. The third time period is July 17, 2016, July 17, 2016. Take all the event sampling, four times a day, one hour each time for 40 days. All event sampling method is used to observing animal behavior, is the expression of function and behavior characteristics, according to the behavior of animals in a certain environment conditions of movement and posture for rapid identification and record. 


\section{Research Results}

\section{A.Motor Behavior}

The movement behavior of Cybister japonicus Sharp is mainly swimming. In addition to ventilation, the Cybister japonicus Sharp planthopper will rise to the surface of the water, most of the time in the water, under water or under water. These include gliding, swimming, swimming, swimming, sinking, floating and backstroke.

Glide refers to the inertia and water flow of the Cybister japonicus Sharp, which can be moved forward without the movement of the swimming feet. Move means that the Cybister japonicus Sharp can move forward with the help of a pair of swimming feet. It is the most common form of behavior and the most important form of swimming. Fast Swim refers to the Fast and Fast speed of the Cybister japonicus Sharp, accompanied by large hind legs and high frequency of swimming. The movement of the ticks is usually irregular and the frequency of motion is very fast.

Swim upside-down means after swimming to a certain layer of water, the head of the Cybister japonicus Sharpreaches back to the surface of the water. There is less chance of this happening. Swim - down refers to the head of the Cybister japonicus Sharp when it reaches a certain layer of water and then returns to the water. There is less chance of this happening. Sink refers to the movement of the Cybister japonicus Sharp, which does not move, and does not use any movement to maintain the original position, which is displaced by water under the action of gravity. Float Upward refers to the movement of the Cybister japonicus Sharp, which is not moved by any movement, so as to maintain the original posture, which is raised by a certain water layer or water to the surface of the water. Backstroke refers to the Cybister japonicus Sharpin's process of swimming, due to hit each other or obstructions, or because of fast turning and turning, the front up to swim, a very short time, one moment over normal winding. There is little chance of it. In addition, the Cybister japonicus Sharp maintains a certain position in the water, and the relative displacement does not change with a particular movement, namely, Immobility. Includes suspension, floating and reclining. Suspension refers to the behavior of the whole body of a Cybister japonicus Sharp to stay in the water and stay in the water. There are two kinds of status: one is normal posture and the back is downward. The second is to use the foot to cling to the stick shape attachment, the abdomen up and backward position to stay in the water layer.

Floating refers to the surface or near surface,Cybister japonicus Sharp's body remained stationary, the body tilted or tail above the water, either on the back of the uplift of above the water, to go with the flow. The forefoot and midfoot of the Cybister japonicus Sharp occasionally reach out, presenting a certain Angle and form with the body vertical axis of the longlice, but most of the time is located below the body's abdomen, which is not seen in the view. The hind legs of the Cybister japonicus Sharp at the time of floating are not moving, and will present different angles and forms on the water surface.

Repose refers to the screen before use, the foot of tarsus joint attached with a foot in the bottom or just tarsus stick attached to the bottom, the abdomen on the bottom stop quietly in the bottom, then presents such as floating various states, sometimes spanning a foot or before, in the foot support in the bottom, the body lift the left bottom, now known as the suspension is more appropriate.

\section{B.Feeding Behavior}

The Cybister japonicus Sharp is a carnivorous animal. The method of feeding is divided into two forms: static feeding and exercise ingestion. When feeding, will use the head two tentacles "temptation" food, and then suddenly with large jaws tightly bite of food, and before the hocks foot hold food, two tentacles keep swinging simultaneously feeding.

Still Feeding refers to the fact that the Cybister japonicus Sharp is used to catch food in front of the foot of the foot before and after the foot of the foot, and then it will be eaten quietly on the water or in the water, and the hind legs will also present a certain Angle. Motion Feeding refers to the fact that the Cybister japonicus Sharp uses its antennae to oscillate around the food, before and after the food is held in front of the foot, and dragging food around the food. Sometimes the food is dragged to the bottom to feed, sometimes towing the food to a certain place, resting in the water for a few 
seconds, then feeding, then continuing to drag the food back and forth in the water. In either form, when the planthopper is full, it leaves the food, stimulates it, and does not feed, which is the end of the feeding behavior. According to the observation of Cybister japonicus Sharp declined significantly below $16^{\circ} \mathrm{C}$ feeding frequency, basic not feeding $10^{\circ} \mathrm{C}, 6^{\circ} \mathrm{C}$ below into hibernation.

\section{C.Reproductive Behavior}

The sexual maturation of the Cybister japonicus Sharp and begins to reproduce. The whole process of reproductive Mating involves Chasing , Clasp and Mating. Reached sexual maturity males yellow edge water beetle, through swimming fast catch up with the one in front of it, has reached sexual maturity female after screen, can't wait to climb back to the female screen body, with its front feet tarsus base swollen sucker adsorption in female screen smooth on both sides of the front of the namib desert, the foot also support on the female of, the lower abdomen on the female spanning back, the hind legs to separate. The hind legs of the female are also separated from the sides. In the male, the female, the male, holds the female and lays the foundation for mating. This process takes 4 minutes. After successful male spanning penis under bending and insert in the female genital tract, mating, the two mate in the water quietly after 19 minutes and 47 seconds, a touch screen walk the mating of the screen, they swim a few over half back states continue to mating, 1 minute 43 seconds after swimming again a few times, continue to mating half back status, 29 points after 17 seconds over to swim to the bottom, in normal position to continue to mate, up or down a few seconds to sink after 2 minutes, still half a minute swim twice and sank to the bottom, two points after swimming 3-4 seconds and sank to the bottom, and then turn to swim up and down for 5 seconds, floating in the water swimming not continue to mate, after a while the male diving beetle driving a few female swimming diving beetle to continue after mating, 4 minutes after mating in the screen due to interference suddenly swim under the two continue on until the end of the mating surface mating two separate. During the entire mating process, the two of them are always handed over and never separated. The mating time lasts one hour and one minute.

\section{D.Eliminative Behavior}

The excretion behaves as a jet.The Cybister japonicus Sharp through the malpighian tube life in intestinal metabolism of waste discharge to bowel, after swimming in a state of spanning the metabolic waste with waste from the anus into in vitro, it looks like inkjet.

Through a systematic and long-term observation, this study constructed the Cybister japonicus Sharp's artificial rearing environment spanning the behavior of the spectrum, the system can not only carry out water beetle behavior research, and for the Cybister japonicus Sharp's artificial breeding research to provide certain reference. The behavior spectrum of the Cybister japonicus Sharp is as follows:

Table 1 The Behavior Spectrum of Cybister japonicus Sharp under Artificial Feeding Conditions

Type: Motor BehaviorFeeding BehaviorReproductive BehaviorEliminative Behavior

Glide Still Feeding
MoveMotion Feeding
Fast Swim
Swim Upside-downChasin
SinkClasp
Float UpwardMating
Backstroke
Hang
Float
ReposeEject




\section{Conclusions and Discussions}

The behavior of the Cybister japonicus Sharp was divided into: motor behavior; Feeding behavior; Reproductive behavior; There are four broad categories of excretion. The form of motor behavior is: gliding, swimming, rapid swimming, backward swimming, sinking, floating, backstroke and special motor behaviors, namely, static behaviors, including suspension, floating and reclining; The behavior of feeding behavior is: static feeding, exercise ingestion; The form of reproductive behavior is: chasing, holding and mating; The excretory behavior forms as ejection. The establishment of behavior spectrum is an important subject of the research on the behavior of insects. It is also a starting point for the study of the regulation behavior of an insect and its adaptation to the environment. Due to constraints, so far, the author by observing the behavior of just the Cybister japonicus Sharp is divided into four categories above, behavioral research for the Cybister japonicus Sharps only do the preliminary work, has yet to be further explored.

The study of the Cybister japonicus Sharp is relatively narrow in the environment, although it has a certain richness in its feeding environment. It also leads to artificial rearing environment the behavior of the income spectrum than the wild population behavior are absent, such as under the condition of artificial feeding, not on screen reproductive Settings link behavior lack of biological environment caused by laying eggs. Also, artificial feeding and field under the condition of the natural environment by the results of comparison, can be found under the condition of artificial feeding, yCybister japonicus Sharp's behavior differences, to understand the Cybister japonicus Sharp under different living conditions, behavior expressed by the elastic.

Study the behavior of animals is not only laid a scientific basis for prevention and control of harmful animals, but also for the use of a variety of beneficial animals and the protection of the rare and endangered animals provide a broad outlook, all these work will be built on the basis of animal behavior study. According to research on some beneficial animal behavior, the utilization range of these animals has been greatly improved. The study of animal behavior is widely applied in animal husbandry and aquaculture sectors to improve economic efficiency and efficiency. In addition, the study of animal behavior also promotes the development of bionics, physiology, psychology, genetics, evolution, taxonomy and ecology, which has an inestimable theoretical significance.

\section{Acknowledgments}

This work was supported by the Key projects of science and technology development plan of Jilin Province,No. 20050216-4、No. 20060907-02 and No. 20130303056NY in part. The author would also like to thank the reviewers for their corrections and helpful suggestion.

\section{References}

[1]Li Chunsheng, Zhou Jingping, Lin Xiumei. Study on the Biological Learning and Artificial Feeding Techniques of Cybister Japonicus Sharp [J].Journal of Baicheng Normal University, 2010(03);

[2]Wei Peilong,Yang Jingyuan,Bao Weidong.Social Behavior Spectrum of the Golden Monkey Feeding Group in Shennongjia [J]. Journal of Biology, 2009(06;

[3]WangXiaoling,Fang Jiao,Wang Lina. Study on Morphology and Structure of Cybister Japonicus Sharp[J]. Journal of Baicheng Normal University,2015(35);

[4]Xiao Jianqiang,Wang Ding.The Construction of Artificial Breeding Environment, The Behavior of the Spectrum of the Yangtze Finless Porpoise [J].Acta Hydrobiologica Sinica ,2005(03);

[5]Wei Chaoming,Kong Guangyao,Guo Lijuan.Preliminary Establishment of Behavioral Spectrum of Housefly[J].Journal of Shaanxi Normal University ,2006(03);

[6]Tian Li,Zhou Caiquan,Wu Kongju.The Behavior Spectrum of Captive Gold Leopard [J].Sichuan Animal ,2009(01). 